\title{
Rotavirus gastroenteritis-associated urinary tract calculus in an infant
}

\author{
Kiwako Tsukida, Masahide Goto, Naoki Yamaguchi, Tomoyuki Imagawa, Daisuke Tamura, \\ Takanori Yamagata \\ Department of Pediatrics, Jichi Medical University, Shimotsuke, Tochigi, Japan. E-mail: mgoto@jichi.ac.jp \\ Received: 24th October 2017, Accepted: 9th December 2017
}

\begin{abstract}
SUMMARY: Tsukida K, Goto M, Yamaguchi N, Imagawa T, Tamura D, Yamagata T. Rotavirus gastroenteritis-associated urinary tract calculus in an infant. Turk
\end{abstract} J Pediatr 2018; 60: 769-770.

Rotavirus gastroenteritis a severe viral gastroenteritis that occasionally causes post-renal failure with urinary tract calculus. A 15-month-old boy with rotavirus gastroenteritis suffered from pre- and post-renal dysfunction due to dehydration and urinary obstruction, respectively. Careful evaluations using abdominal ultrasound and cautious fluid replacement with urine alkalization led to an improvement in the pre- and post-renal dysfunction.

Key words: gastroenteritis, urinary calculi, renal failure, metabolic acidosis.

Rotavirus gastroenteritis, which develops in infants and young children, is severe and often results in dehydration, leading to pre-renal failure. Rotavirus gastroenteritis-induced acute calculus has been reported to lead to post-renal failure due to bilateral urinary obstruction. ${ }^{1}$ In most cases, renal dysfunction develops approximately 1 week after the onset of gastroenteritis, and severe cases require dialysis and a renal fistula.3,5.

Herein, we report the case of an infant with preand post-renal dysfunction due to dehydration and urinary obstruction, respectively.

\section{Case Report}

A 15-month-old boy was being medicated with furosemide and spironolactone for chronic heart failure and aortic valve stenosis. He presented with a 3-day history of fever, frequent vomiting, and diarrhea. Upon physical examination, he exhibited an irritable mood, poor activity, dry mouth and skin, prolonged capillary refill time, and a $7 \%$ decrease in body weight over the 3 days. The laboratory findings were as follows: a blood $\mathrm{pH}$ of $7.32, \mathrm{HCO}_{3}{ }^{-}$level of $11.7 \mathrm{mmol} / \mathrm{L}$, base excess level of $-12.2 \mathrm{mmol} / \mathrm{LM}$, blood urea nitrogen level of $21 \mathrm{mg} / \mathrm{dl}$ (normal, $11 \mathrm{mg} / \mathrm{dl}$ ), creatinine level of $0.3 \mathrm{mg} / \mathrm{dl}$ (normal, $0.2 \mathrm{mg}$ / $\mathrm{dl}$ ), uric acid level of $15.6 \mathrm{mg} / \mathrm{dl}$ (normal, 5.4 $\mathrm{mg} / \mathrm{dl}$ ), urinary specific gravity of 1.032 , a urine $\mathrm{pH}$ of 5.5, and a positive stool rotavirus antigen test. These laboratory values were diagnostic of metabolic acidosis, mild renal dysfunction, and hyperuricemia. He was hospitalized for moderate dehydration and anuria caused by rotavirus gastroenteritis. A drip infusion did not facilitate urine formation. An abdominal ultrasound showed a lack of urine in the bladder, urinary tract calculus with acoustic shadows, and hydronephrosis (grade 2) of both kidneys. Postrenal failure caused by urolithiasis associated with rotavirus gastroenteritis was suspected. Urination resumed after urine alkalinization with sodium bicarbonate and continued fluid infusion. Repeat abdominal ultrasound confirmed precipitates in the bladder (Fig. 1A), and a large number of amorphous urate crystals were excreted (Fig. 1B). He was capable of oral intake and was discharged after recovery from hydronephrosis, as determined based on ultrasound findings. Written informed consent was obtained from the patient's family.

\section{Discussion}

In the present case, pre-renal dysfunction was caused by a dehydrated state, which also caused marked weight loss, and post-renal dysfunction was caused by urinary obstruction due to uric acid crystals. Both pathological conditions were improved with careful fluid replacement and urine alkalization. On the third day of onset, body weight decreased by $7 \%$, rapid dehydration led to pre-renal dysfunction, and uric acid levels increased to $15.6 \mathrm{mg} / \mathrm{dl}$. Under the conditions 

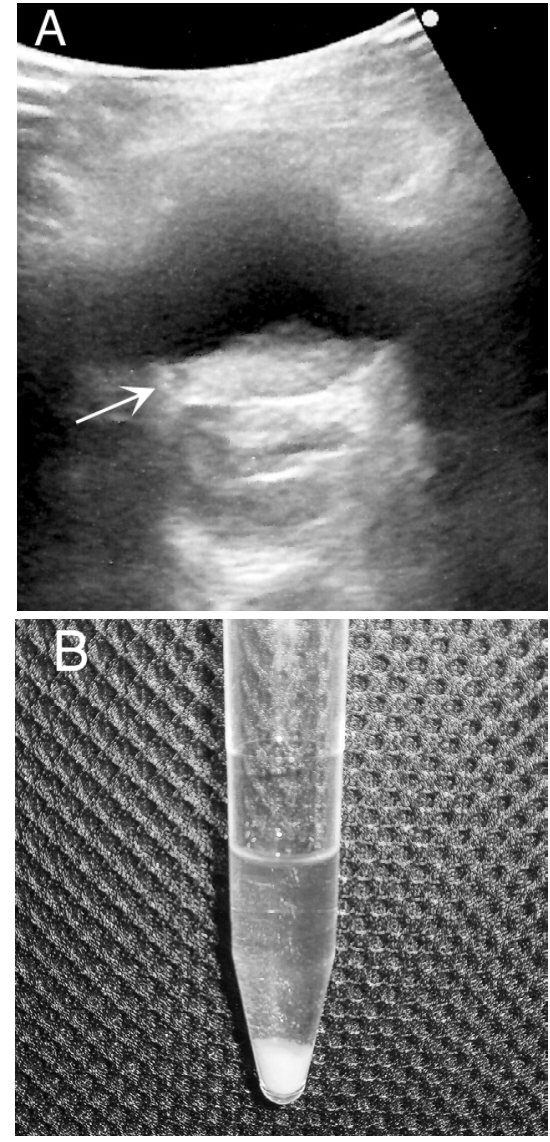

Fig. 1. (A) Ultrasound showing precipitates (arrow) with acoustic shadows in the bladder. (B) A large amount of amorphous urate crystals present in the urine.

of urine acidosis ( $\mathrm{pH}$ 5.5) and hyperuricemia, urine crystals were made from acidic urine, causing post-renal dysfunction. This dehydrated condition was exacerbated by the addition of diuretics (for heart disease) to the natural course of rotavirus gastroenteritis. In cases of suspected complications of pre- and post-renal failure, there is a risk of exacerbating post-renal dysfunction with only fluid replacement therapy, which is a treatment for pre-renal failure. Alkalization of the urine is necessary for preventing and dissolving calculi, in addition to carefully confirming the extent of urinary tract obstruction through an abdominal ultrasound.

Rotavirus gastroenteritis has been reported to result in post-renal failure due to the development of ammonium urate crystals and in pre-renal failure due to dehydration. ${ }^{2,3}$ Most cases of renal dysfunction occur approximately 1 week after the onset of gastroenteritis, during the gastroenteritis recovery period. However, when dehydration rapidly progresses from the early stages and the urine $\mathrm{pH}$ exhibits an acidic trend, post-renal failure may result from urinary obstruction due to the development of uric acid crystals and calculi.

The pathophysiology underlying the formation of urinary stones associated with rotavirus gastroenteritis remains unclear, but the pathological mechanism is considered to be as follows. ${ }^{3}$ First, when dehydration occurs due to gastroenteritis, increased uric acid levels in the blood lead to an increase in uric acid levels in the urine. In addition, electrolytes and ions, including carbonic acid, are lost due to severe diarrhea, resulting in metabolic acidosis. Furthermore, as electrolyte loss continues and urinary sodium excretion decreases, the binding of uric acid and ammonia ions is induced, thereby promoting acid ammonium urate calculus formation. After 5 or 6 days of metabolic acidosis, the kidneys secrete ammonia to buffer the increased $\mathrm{H}^{+}$secretion ${ }^{4}$, leading to the formation of ammonium acid urate calculi under conditions of uric acid oversaturation. Conversely, during the early stages of rotavirus gastroenteritis (day 1 or 2), little ammonia is secreted into the urine; however, when uric acid oversaturation develops, urate calculi are formed, ${ }^{3}$ similar to the present case.

When reduced urinary volume caused by rotavirus gastroenteritis is noted, abdominal ultrasound evaluation from the kidney to the bladder should be performed to detect the pre-renal disorder of dehydration and post-renal disorder of urinary obstruction. For the treatment of coexisting pre- and post-renal dysfunction, cautious fluid replacement to improve metabolic acidosis and urine alkalization should be considered.

\section{REFERENCES}

1. Dennehy PH. Rotavirus infection: A disease of the past? Infect Dis Clin North Am 2015; 29: 617-635.

2. Ashida A, Fujieda M, Ohta K, et al. Clinical characteristics of obstructive uropathy associated with rotavirus gastroenteritis in Japan. Clin Nephrol 2012; 77: 49-54.

3. Shirasu A, Ashida A, Matsumura H, Nakakura H, Tamai $\mathrm{H}$. Clinical characteristics of rotavirus gastroenteritis with urinary crystals. Pediatr Int 2015; 57: 917-921.

4. Tizianello A, Deferrari G, Garibotto G, Robaudo C, Acquarone N, Ghiggeri GM. Renal ammoniogenesis in an early stage of metabolic acidosis in man. J Clin Invest 1982; 69: 240-250.

5. Kira S, Mitsui T, Zakoji $\mathrm{H}$, et al. Postrenal failure due to urinary stones associated with acute viral gastroenteritis: Three case reports. Case Rep Urol 2016; 2016: 1375923. 


\title{
A child with idiopathic facial aseptic granuloma
}

\author{
Müzeyyen Gönül ${ }^{1}$, Şensu Tufan ${ }^{1}$, Deniz Ateş Özdemir ${ }^{2}$, Fatma Aksoy-Khurami² \\ Departments of ${ }^{1}$ Dermatology and ${ }^{2}$ Pathology, University of Health Sciences Diskapi Ylldrrm Beyazit Training and Research \\ Hospital, Ankara, Turkey. E-mail: sensutufan@hotmail.com \\ Received: 24th September 2017, Revised: 26th November 2017, Accepted: 24th December 2017
}

Idiopathic facial aseptic granuloma (IFAG) is a rare skin disorder characterized by one or few asymptomatic red or purplish papules or nodules that occur specifically on cheeks or eyelids of children. ${ }^{1,2}$ The etiology of IFAG is unclear. $^{2}$ The disease heals spontaneously within a few months, up to 1 year, without a scar. ${ }^{1}$ We present a peculiar case of IFAG with multiple nodules associated with an eyelid chalazion in a 3-year-old boy.

A 3-year-old boy presented to our outpatient clinic with papules and nodules located on his right cheek. The lesions occurred on his right cheek 9 months before he was presented and did not respond to many topical and systemic antibiotics, topical steroids and intramuscular triamcinolone acetonid therapies. His parents defined that a hordeolum recurred two times on the right eyelid. There was no history of bleeding, purulent discharge or ulceration and trauma or insect bite before it. Dermatological examination showed a few about $1 \mathrm{~cm}$ sized, painless, separate, erythematous-violaceous nodules located on the right cheek and a chalazion of the right upper eyelid (Fig. 1). There were no accompanying blackheads, telangiectasia or flushing. Ultrasonographic examination of the nodules on the right cheek revealed a well-limited, hypoechoic lesion without calcium deposits. Histopathological examination showed dermal inflammation with histiocytes, scattered giant cells, lymphocytes, neutrophils extending into deep dermis (Fig. 2). Leishmania amastigotes by Giemsa staining and demodex mites could not be seen in histopathological examination. No specific microorganism was detected with EhrlichZiehl-Neelsen, Periodic Asid Schiff and Gram staining. IFAG was diagnosed on the basis of medical history, physical examination, ultrasound and pathological findings. When parents were informed about the illness, they preferred untreated follow-up. After six months, the lesions gradually and spontaneously healed without scars (Fig. 3).

IFAG characterized by generally asymptomatic facial papules or nodules originally was described as "pyodermite froide" because of clinical similarity to pyoderma or cyctic lesions. ${ }^{1,3}$ It is usually located within a triangle limited by the external corner of the orbit, the labial angle, and the ear lobe. ${ }^{2}$ Blackheads and telengectasia are not associated with this disease. ${ }^{3}$ Satta et al. ${ }^{3}$ reported that mean onset age of the disorder was 12 months. The disorder was seen slightly higher in girls. ${ }^{3}$ In most cases of IFAG it is mostly solitary and multiple lesions are rarely seen. ${ }^{3}$ Our patient was a 3 year-old boy and he had 4 nodules.

The etiology of IFAG is unclear. ${ }^{2}$ However, there have been several hypothesis and one of them is a possible granulomatous response to an uncertain embryologic remnant. ${ }^{4}$ Another hypotesis is that IFAG might be a granulomatous form of rosacea because some cutaneous findings are similar to granulomatous rosacea (GR) and association with facial telengiectasias, recurrent chalazions and conjunctivitis. ${ }^{2}$ Prey et $a .^{5}$ reported that the children with IFAG had a greater risk of developing rosacea, especially ocular rosacea, and prevalence of rosacea was $42.1 \%$ in their IFAG population. But some authors think that IFAG is a different disease from GR because it has tendency of spontaneous regression and more diffuse and deeper infiltration than GR. ${ }^{3}$ He has followed for his recurrent chalazion.

The differential diagnoses of IFAG include much dermatological entity such as nodular infantile acne, pediatric rosacea, pyoderma, dermoid cyst, leishmania, xanthogranulomas, tuberculids, papulonodular skin lesions of Crohn's disease, vascular malformations and insect bite.,2,3 Skin ultrasound may be useful for diagnosis and it shows a well-limited, hypoechoic lesion 


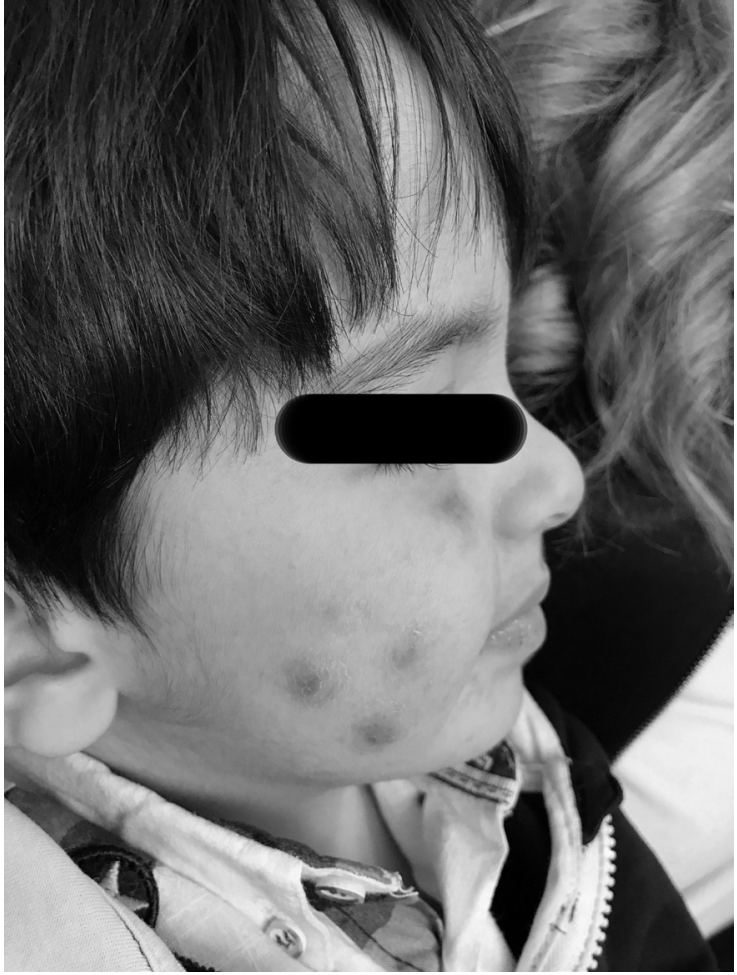

Fig. 1. A few, about $1 \mathrm{~cm}$ sized, painless, separate, erythematous-violaceous nodules located on the right cheek and a chalazion of the right upper eyelid.

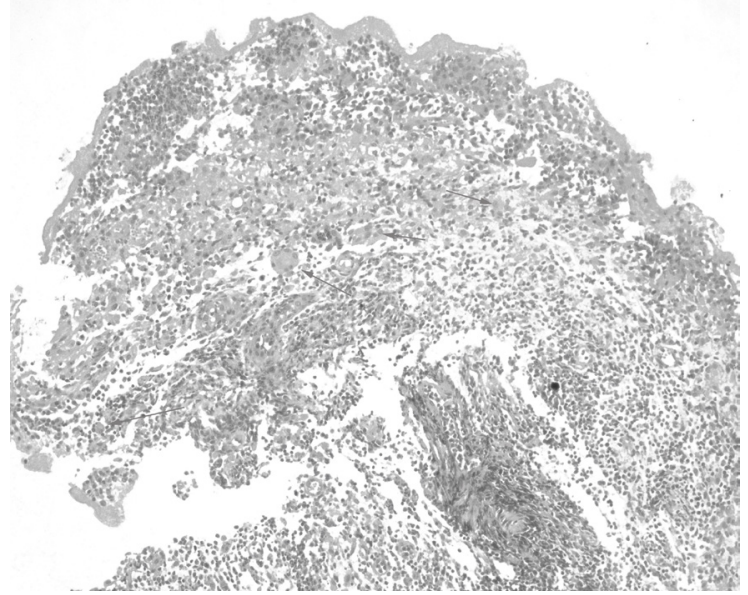

Fig. 2. Polymorphic inflammatory infiltrate which is composed of histiocytes, lymphocytes, neutrophils and eosinophils (giant cells were marked with arrows) (H\&E X 100)

without calcium deposits. ${ }^{1}$ Cultures obtained from purulent material produce negative results for bacteria, fungi except superinfection. ${ }^{4}$ Histopathological examination of IFAGs shows diffuse infiltrate of histiocytices, giant cells, lymphocytes and neutrophils in the deep dermis. ${ }^{3}$ Clinical, histopathological and

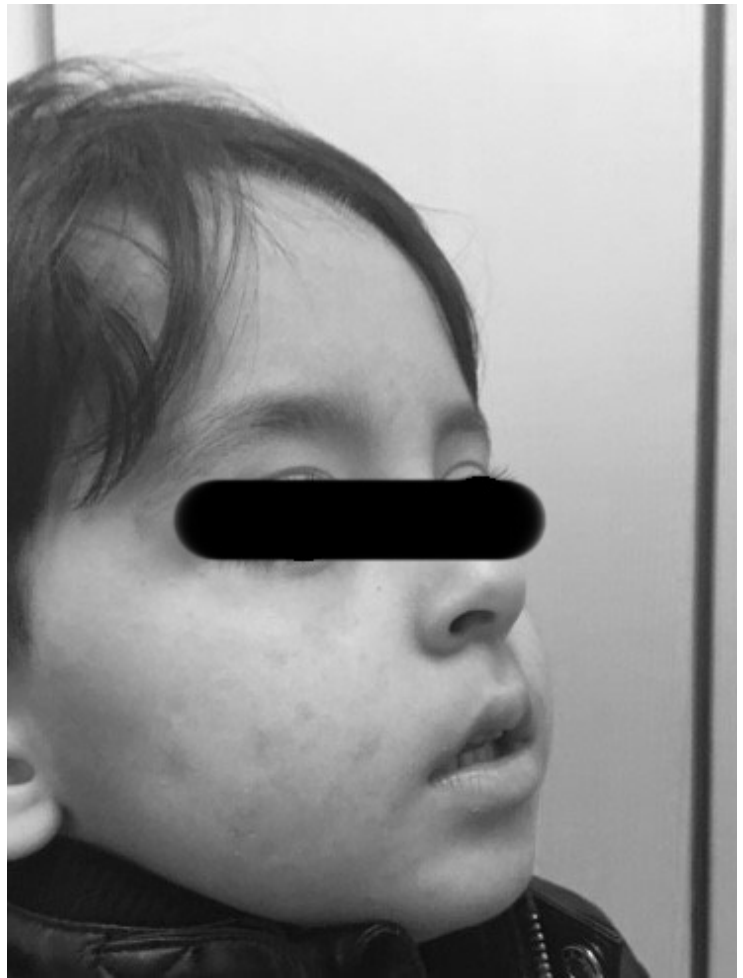

Fig. 3. After six months, the lesions spontaneously healed without scars

ultrasounographic findings of our case were compatible with IFAG.

The diagnosis of IFAG can be missed as in our case because of its rarity. The dermatologist should keep this disease in mind in a child with a nodular lesion on the face.

Key words: aseptic granuloma, children, face, nodules.

\section{REFERENCES}

1. Zitelli KB, Sheil AT, Fleck R, Schwentker A, Lucky AW. Idiopathic facial aseptic -granuloma: Review of an evolving clinical entity. Pediatr Dermatol 2015; 32: e136-e139.

2. Neri I, Raone B, Dondi A, Misciali C, Patrizi A. Should idiopathic facial aseptic granuloma be considered granulomatous rosacea? Report of three pediatric cases. Pediatr Dermatol 2013; 30: 109-111.

3. Satta R, Montesu MA, Biondi G, Lissia A. Idiopathic facial aseptic granuloma: Case report and literature review. Int J Dermatol 2016; 55: 1381-1387.

4. Boralevi F, Léauté-Labrèze $\mathrm{C}$, Lepreux $\mathrm{S}$, et al; Groupe de Recherche Clinique en Dermatologie Pédiatrique. Idiopathic facial aseptic granuloma: A multicentre prospective study of 30 cases. Br J Dermatol 2007; 156: 705-708. 
5. Prey S, Ezzedine K, Mazereeuw-Hautier J, et al.; Groupe de Recherche Clinique en Dermatologie Pédiatrique. IFAG and childhood rosacea: A possible link? Pediatr Dermatol 2013; 30: 429-432. 